\title{
Mejoramiento de procesos de manufactura utilizando Kanban
}

\author{
Martin Darío Arango Serna * \\ Luis Felipe Campuzano Zapata ** \\ Julián Andrés Zapata Cortes ${ }^{* * *}$
}

Recibido: 13/08/2014 • Aceptado: 11/05/2015

\section{Resumen}

Este artículo presenta la aplicación de la metodología Kanban y el análisis del efecto que puede generar en una empresa de fabricación de transformadores de distribución. Mediante la aplicación de la metodología propuesta es posible mejorar la programación de la producción, con el objetivo de reducir la cantidad de producto en proceso que no es utilizado, de forma que se reduzca el inventario. Para analizar el efecto de aplicar la metodología Kanban en la empresa, se utilizo la técnica de simulación, para lo cual se modelizan el proceso actual y el propuesto con las reglas de dicha metodología. A partir de los resultados que arrojan dichas modelizaciones, se observa que existe un mejoramiento en las líneas de producción cuando se utiliza la metodología Kanban.

Palabras clave: Mejoramiento de procesos, Kanban, simulación, manufactura, optimización de procesos productivos.

\footnotetext{
Doctor en Ingeniería Industrial. Profesor titular. Departamento Ingeniería de la Organización., Universidad Nacional de Colombia, Sede Medellín, Director Grupo de I+D+I Logística Industrial - Organizacional -GICO-. Cra 80 N. 65 -223. Bloque M8B-211. Tel: 4255215. mdarango@unal.edu.co

** Magíster en Ingeniería Administrativa. Ingeniero de Producción. Rymel S.A.S. 1fcampuz@unal.edu.co

*** Magíster en Ingeniería Administrativa. Estudiante de Doctorado. Investigador Grupo GICO, Universidad Nacional de Colombia, Sede Medellín, Facultad de Minas. jazapat1@unal.edu.co
} 


\title{
Manufacturing process improvement using the Kanban
}

\begin{abstract}
This paper presents the application of the Kanban methodology and analysis of the effect that this can generate in a manufacturing of distribution transformers. By applying the proposed methodology can be improved production scheduling, with the aim of reducing the amount of product in process is not used, so that inventory is reduced. To analyze the effect of applying the Kanban methodology in the company, the simulation technique was used, for which the current process and proposed to the rules of the Kanban methodology is modeled. From these results that yield modeling, it shows that there is an improvement in production lines where kanban methodology is used.
\end{abstract}

Key words: Process improvement, Kanban, simulation, manufacturing, optimization of production processes 


\section{INTRODUCCIÓN}

En la actualidad, la necesidad de producir eficientemente sin causar trastornos ni retrasos en la entrega de una referencia de producto es un factor de suma importancia para las empresas que desean permanecer activas en el mercado, el cual exige respuestas rápidas y cumplimientos en calidad, cantidad y tiempos de entrega. Por lo tanto, la implementación de sistemas de producción más eficientes ha llegado a ser un factor que es crucial para las plantas de manufactura que busquen eficiencia y eficacia en sus procesos.

Existen varias metodologías que conducen al mejoramiento de los procesos, en donde las técnicas japonesas han sido un referente importante, debido a los resultados que estas ofrecen y la amplia bibliografía que se puede encontrar al respecto facilitan su conocimiento y aplicación [1-9]. Estas técnicas japonesas de mejoramiento de procesos son de amplio conocimiento en los entornos industriales y académicos, y se pueden citar, como las más conocidas: Mantenimiento Total de la Producción (TPM), Justo a Tiempo (JIT) y Gestión Total de la Calidad (TQM). Sin embargo, existen otras metodologías que pueden ser aplicadas en áreas de manufactura sin grandes inversiones en dinero, pero que permiten obtener resultados exitosos en la mejora de los procesos, como es el caso de la metodología Kanban.

Raymond [10] define Kanban como "un dispositivo de señalización desarrollado por Toyota para el movimiento de partes en un sistema de producción por demanda, generalmente mediante el uso de una tarjeta física. El objetivo de Kanban es minimizar el TEP (Trabajo en progreso), o el stock entre los procesos. Para lograr esto, Kanban se asegura que el proceso superior produzca partes, sólo si el proceso inferior las necesita; Por demanda, se entiende que los trabajadores del proceso inferior consumen las partes que necesitan de los procesos superiores" [10].

En la investigación aplicada, aquí presentada, se realiza una simulación con dos ambientes de trabajo utilizados: con y sin implementar la metodología Kanban. Utilizando el software SIMUL8 [11] como herramienta de simulación, se presenta el proceso de fabricación de transformadores para ambos ambientes de trabajo. Posteriormente, se presentan los resultados obtenidos, comparativos de ambos ambientes.

\section{METODOLOGÍA KANBAN}

Existen en la literatura múltiples definiciones sobre la metodología Kanban. Para fines del desarrollo de este artículo, se considera la definición presentada por Acevedo et al. [12] la cual establece que Kanban es "una técnica de gestión de producción basada en un sistema pull (halar) que se fundamentan en la autogestión de los procesos, eli- 
minando la programación centralizada. Se produce y transporta lo que se demanda en los procesos consumidores, manteniendo en rotación sólo aquellas cantidades que garantizan la continuidad del consumo. Cuando se interrumpe el consumo se detiene la producción. Es una herramienta para conseguir la producción Justo a tiempo -JIT-”."

Según Acevedo et al. [12], con Kanban se reduce el sistema de información, ya que no se requiere elaborar el plan detallado para la subdivisión productiva o para cada proceso. En su lugar, basta con informar a la línea de montaje final o proceso terminal [12]. Esto puede expresarse como: "El proceso siguiente retira las piezas (trabajo) del proceso anterior".

Por su parte, Geek with Laptop [13] definen la palabra Kanban como parte de las palabras kan (看 ○ カン) que significa visual y ban (板 ○バン) que significa tarjeta o tablero. La idea surge en el seno de la metodología Lean, la cual fue desarrollada por Toyota, para mejorar la producción basándose en técnicas como el justo a tiempo (JIT).

Los principios que se promueven en la metodología Kanban son [14]:

- Calidad perfecta a la primera: Todo lo que se hace se debe intentar hacerlo bien, no rápido, ya que cuesta más tiempo hacer algo rápido y tener que arreglarlo después, que hacerlo bien desde el principio.

- Minimización del despilfarro: Hacer lo justo y necesario, sin entretenerse en otras tareas secundarias o innecesarias (principio YAGNI).

- Mejora continua: Ir mejorando continuamente los desarrollos, según los objetivos a lograr y alcanzar.

- Flexibilidad: Según los faltantes o pendientes se deciden las tareas a realizar. Las tareas entrantes se pueden priorizar y condicionar según las necesidades puntuales.

- Construcción y mantenimiento de una relación a largo plazo con proveedores.

La metodología Kanban está basada en un conjunto de seis reglas, las cuales se presentan en la figura 1. En el desarrollo de este tipo de técnicas, es muy común ver adheridas tarjetas (o simplemente tarjetas de tareas) en un tablero o en una pared, las cuales son conocidas como "Tareas Kanban". 


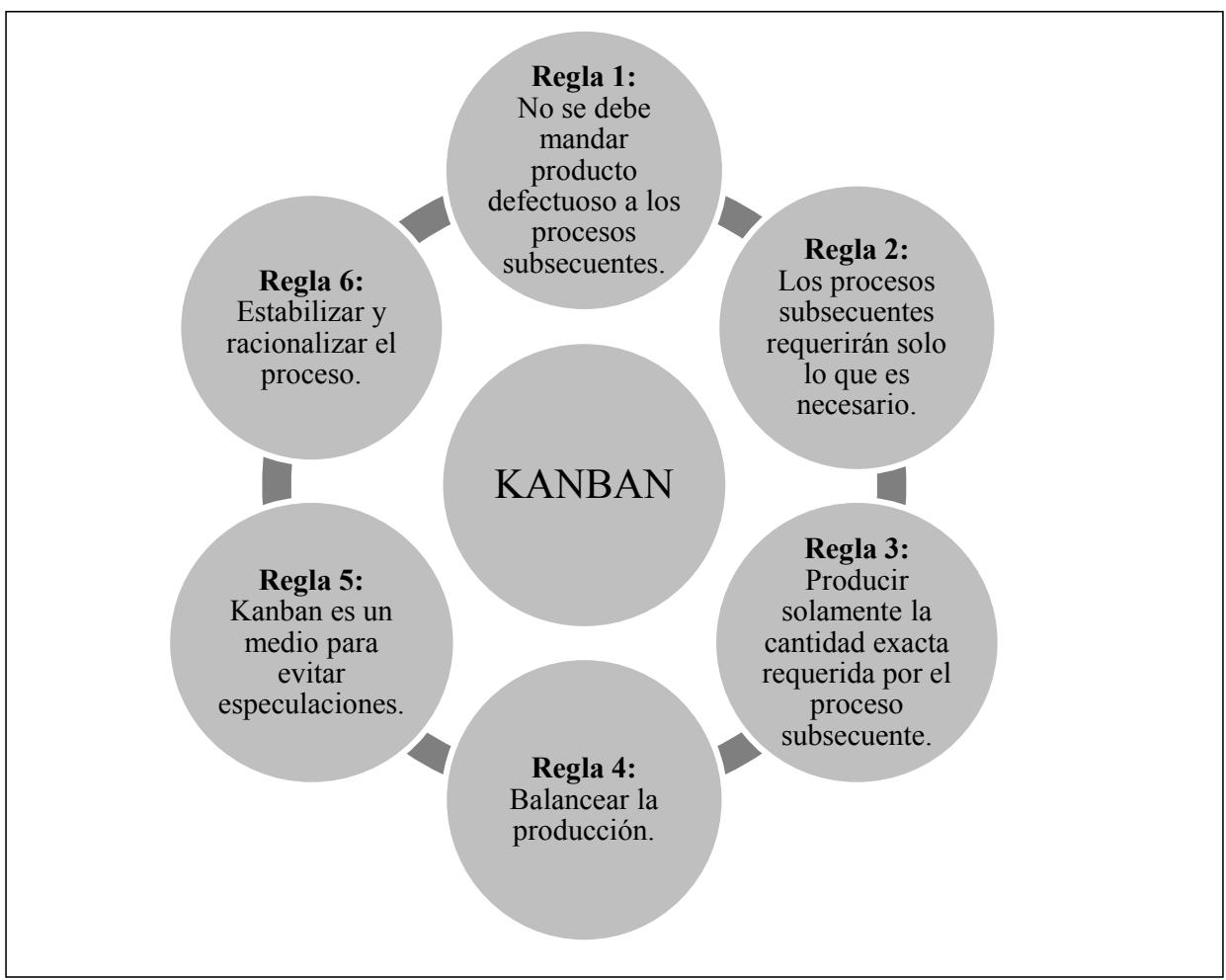

Figura 1. Reglas en las que se basa la metodología Kanban Fuente: elaboración propia

En un proceso de manufactura, los componentes de la referencia de producto a fabricar se distribuyen desde un proceso superior hacia un proceso inferior. Una tarjeta Kanban es el punto de partida de una tarea, en la cual hay información escrita como la identificación, el nombre, el tiempo estimado, el nombre de quien está asignado a la tarea, etc. Cada una de las tarjetas posee un estado (pendiente, en trabajo, terminada) las cuales son compartidas por todo el equipo de trabajo y tienden a minimizar la burocracia dentro del equipo.

Por otra parte, se puede utilizar un sistema Kanban en cascada tradicional pero con flujo, donde es posible separar varios procesos de manufactura y las tarjetas se mueven entre procesos como "diseño", "desarrollo" y "validación". Cada tarjeta Kanban representa un aviso para el sistema, las cuales van avanzando de una a una, logrando así una fase estable y sustentable en el ciclo de vida del producto [10]. Kanban se ha extendido desde los procesos de manufactura a otras áreas, y en algunas empresas se ha posicionado como unos de los sistemas más complejos y eficaces para la mejora de sus procesos de manufactura y administrativos $[10,14]$. 


\section{METODOLOGÍA}

La metodología Kanban es aplicable a entornos repetitivos de manufactura en donde el material fluye en rutas fijas y tasas constantes. Para estos casos existe gran variedad de técnicas que funcionan bajo el mismo esquema en el cual se aplica Kanban. En este artículo se desarrolla la metodología Kanban, pero ajustada al entorno de producción que se tiene en la empresa de estudio, la cual se dedica a la fabricación de transformadores de distribución, con la particularidad de que su plan de producción exige la fabricación de múltiples referencias de transformadores, la materia prima fluye entre los proceso por rutas fijas, y debido a las diferentes referencias que se procesan, los tiempos de las operaciones son variables.

Para realizar el análisis de la metodología Kanban se modelizó la fabricación de un transformador "representativo" de las diferentes referencias que existen en la empresa; este transformador se seleccionó teniendo en cuenta el consumo de materia prima y la intensidad de la mano de obra. Como punto de inicio para la implementación de la metodología Kanban en el proceso de simulación, se utilizo el punto de encuentro o cruce entre los procesos de bobinas y Núcleos en la fabricación de transformadores de potencia. Este es uno de los puntos de mayor importancia, debido al alto volumen de producto en proceso que usualmente se acumula al finalizar en los dos procesos: bobinas y núcleos. Al ser un punto crítico para el control de producto en proceso, se convierte en el principal candidato para analizar la metodología Kanban, esto con el objeto de obtener resultados que puedan ser replicables a los demás puntos de control de producto en proceso dentro de la planta de producción [15].

La modelización presentada en este artículo se centra en los procesos de bobinado y núcleo, en donde se espera establecer por medio de la implementación de Kanban una mejor coordinación y control sobre estos dos procesos, con lo que se busca alcanzar los siguientes objetivos:

- Minimizar el producto en proceso.

- Mejorar la comunicación entre procesos.

- Obtener mejor control sobre los inventarios.

- Prevenir la sobreproducción.

- Incentivar en el personal el mantenimiento de los procedimientos estandarizados.

- Hacer visible el control de flujo de material.

- Establecer una programación que muestre el estado de la producción.

- Mostrar limitaciones que puedan ser direccionadas por Kaizen. 
La implementación de la metodología Kanban se basa en el seguimiento de las seis reglas naturales presentadas en la figura 1, tal como es descrito por Raymond [10] y otros autores. La implementación de la metodología Kanban, según lo establecido por Ballesteros [15], se realiza mediante la ejecución de 4 fases necesarias para su correcta aplicación, las cuales son:

- Fase 1: Entrenar a todo el personal en los principios de Kanban y los beneficios de usarlo.

- Fase 2: Implementar Kanban en los componentes con más problemas para facilitar su manufactura y para resaltar los problemas escondidos. El entrenamiento con el personal continúa en la línea de producción.

- Fase 3: Implementar Kanban en el resto de los componentes. Se deben tomar en cuenta todas las opiniones de los operadores, ya que ellos son los que mejor conocen el sistema. Es importante informarles cuando se va a estar trabajando en su área de responsabilidad.

- Fase 4: Esta es la fase para la revisión del sistema Kanban, los puntos de reorden y los niveles de re-orden. Es importante tomar en cuenta las siguientes recomendaciones para el funcionamiento correcto de este sistema:

a) Ningún trabajo debe ser hecho por fuera de secuencia.

b) Si se encuentra algún problema, notificar al supervisor inmediatamente.

El desempeño del sistema Kanban en la metodología propuesta se evalúa mediante la vigilancia de los siguientes elementos:

- Se siguen las seis reglas de Kanban.

- Los niveles de las áreas de inventario/ TEP (trabajo en proceso) están claramente marcadas.

- Una tarjeta Kanban/ etiquetado/ código de barras en cada contenedor.

- Un inventario exacto.

- Cantidad mínima de TEP (trabajo en progreso) entre las estaciones.

- Capacitación en Kanban a todos los niveles de la planta.

- Kanban establece las prioridades de material del operador.

- $\quad$ Flujo de materiales en PE-PS (primeras entradas - primeras salidas). 
En la literatura especializada y en la industria existe gran cantidad de herramientas que optimizan las funciones de identificación, control y manejo de la información de las materias primas, producto en proceso, producto terminado, entre otros [16-17]. En el proceso de fabricación de transformadores existen varias etapas de horneo que oscilan regularmente entre los 120 y $800 \mathrm{pc}$, dependiendo del proceso de manufactura, por lo cual se recomienda el uso de una tarjeta física, con la que se pueden satisfacer las necesidades de ubicar los lotes y priorizar los pedidos que se lanzan a producción, flexibilizando la posibilidad de ejercer cambios sobre la misma tarjeta, lo cual es muy común en la fase de implementación y adaptación de una metodología de trabajo.

\section{SIMULACIÓN CON LA METODOLOGÍA KANBAN}

Con el objeto de validar los alcances de la metodología Kanban propuesta, se desarrollaron dos modelos de simulación mediante el software SIMUL8. En el primer modelo, como se observa en la figura 2 , se muestra la forma como operan actualmente las líneas de fabricación de bobinado, núcleo y su punto de encuentro para la entrega posterior a la línea de ensamble, armado y conexión. El segundo modelo (figura 3) muestra la misma configuración pero con la implementación de la metodología Kanban propuesta. Posteriormente se evaluarán las salidas del modelo número 1 (Actual) frente a modelo número 2 (Actual + Kanban) y se valora el desempeño de la metodología. En el modelo número 2 , no se modifica ningún parámetro de tasa de producción ni se efectúan cambios sobre las capacidades de producción de los centros de trabajo. El cambio en el modelo número 2 respecto al modelo número 1 solo obedece a la metodología Kanban propuesta, la cual solo incide en la forma como se programa la producción. Las tasas de producción empleadas son supuestas con el objetivo de preservar la privacidad de la empresa en la cual se realizó la investigación aplicada.

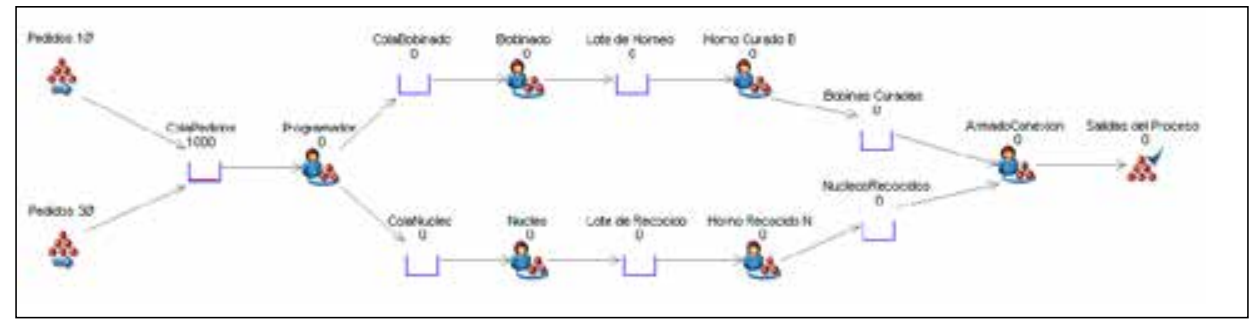

Figura 2. Modelo de simulación \#1

Fuente: elaboración propia 


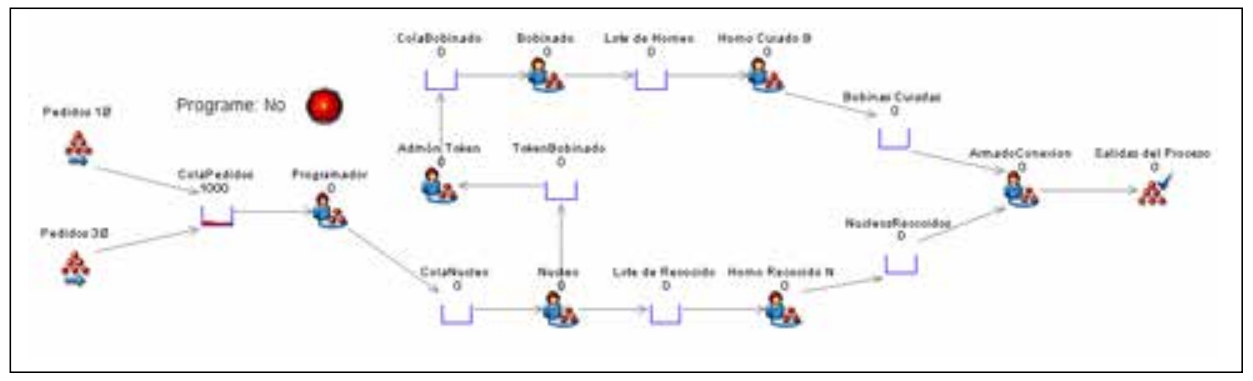

Figura 3. Modelo de simulación \#2.

Fuente: elaboración propia

\section{ANÁLISIS DE RESULTADOS}

En la figura 4, se observa el porcentaje de núcleos que quedan sin bobina para la producción de transformadores de potencia con el método actual, evidenciando un comportamiento altamente variable, lo que permite argumentar sobre la falta de coordinación entre procesos, debido a los altos porcentajes de núcleos sin bobinas. Teniendo en cuenta que los porcentajes de núcleos sin bobinas aplicando la metodología Kanban son bajos, se evidencia que la utilización de esta presenta una mejora en la rotación de producto en proceso, contribuyendo a tener el mínimo de unidades en cola y en inventario. En la tabla 1 se presenta la media y la desviación estándar del porcentaje de núcleos sin bobina, para el funcionamiento actual y con la implementación de la metodología camban.

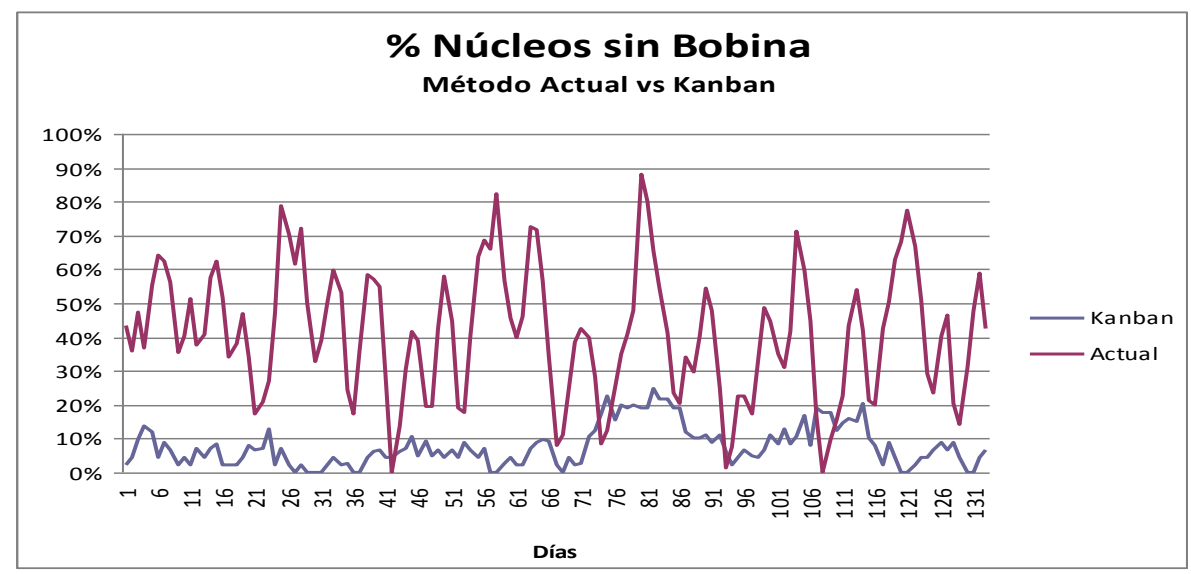

Figura 4. Porcentaje de núcleos recocidos sin bobina.

Fuente: elaboración propia 
Tabla 1. Valor medio y variabilidad del número de núcleos sin bobina.

\begin{tabular}{|l|c|c|}
\hline \multicolumn{1}{|c|}{ Proceso } & Media & Desviación \\
\hline Actual & $40.7 \%$ & $17.5 \%$ \\
\hline Con Kanban & $6,9 \%$ & $5.2 \%$ \\
\hline Diferencia & $33.8 \%$ & $12.3 \%$ \\
\hline
\end{tabular}

Fuente: elaboración propia

En la figura 5 se puede observar que con el método actual se mantiene un porcentaje muy alto de bobinas sin núcleo, y no se observan caídas súbitas de este porcentaje tal y como ocurre en el figura 4, lo se debe a que la forma de programar la producción para el proceso de bobinado y núcleo es independiente al tiempo de ciclo de ambos procesos, programándolos en el mismo instante de tiempo. Por lo anterior se incurre en un tiempo de espera en la cola de bobinas, ya que su tiempo de ciclo de fabricación es inferior al de núcleos.

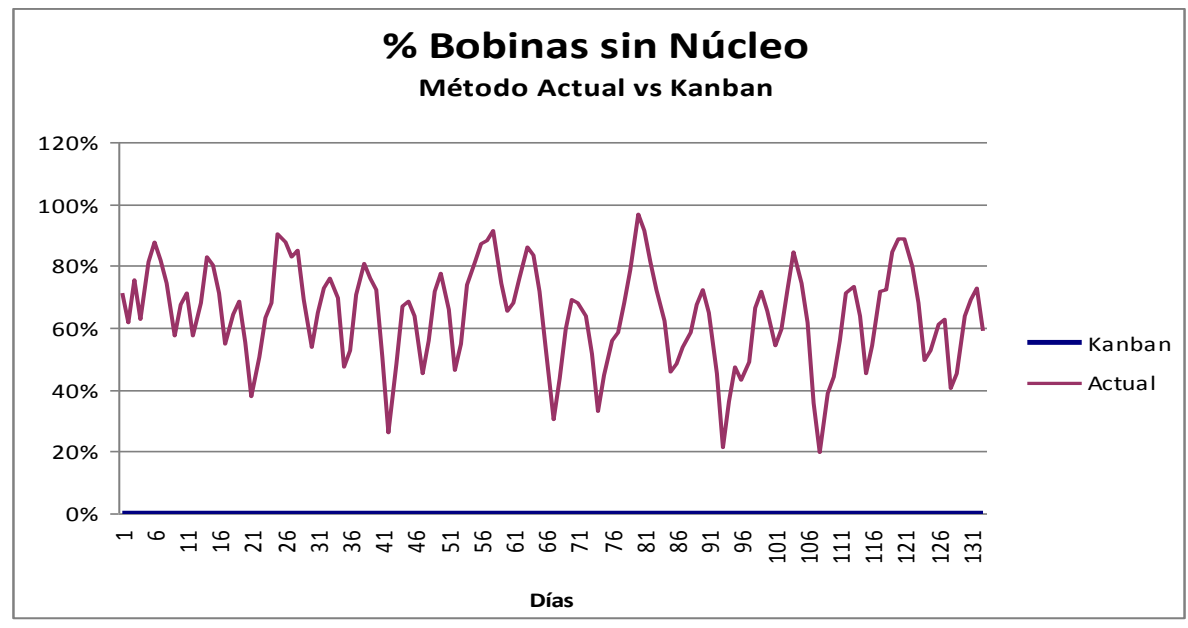

Figura 5. Porcentaje de bobinas sin núcleo.

Fuente: elaboración propia

Con la metodología Kanban propuesta, el porcentaje de bobinas sin núcleo es muy bajo. Esto se debe a las reglas que establece la metodología Kanban en su implementación, en la cual el lote de producción que se programa en el día X para la sección de bobinado es el lote que se cargó en el horno de recocido en el día X-1. Esta regla garantiza que las bobinas a procesar sí tengan núcleo recocido al finalizar su proceso. Con la regla anterior se produce un resultado que es correspondiente a un justo a tiempo en la línea de armado y conexión, con lo cual se genera una disminución en los niveles de inventario de producto en proceso. 
En la figura 6 se muestra que en el método actual las órdenes de producción son empaquetadas en grandes lotes y enviadas a ambos procesos en el mismo instante de tiempo, lo que genera poca coordinación entre procesos para la ejecución de las órdenes. Lo anterior se presenta, debido a que cada Centro de Trabajo ejecuta las órdenes con prioridades diferentes, es decir, en el Centro de Trabajo de núcleo se prefiere ejecutar las órdenes por tipo de referencia con el objeto de minimizar tiempos de montaje y en bobinado se trabaja basándose en la prioridad de los pedidos.

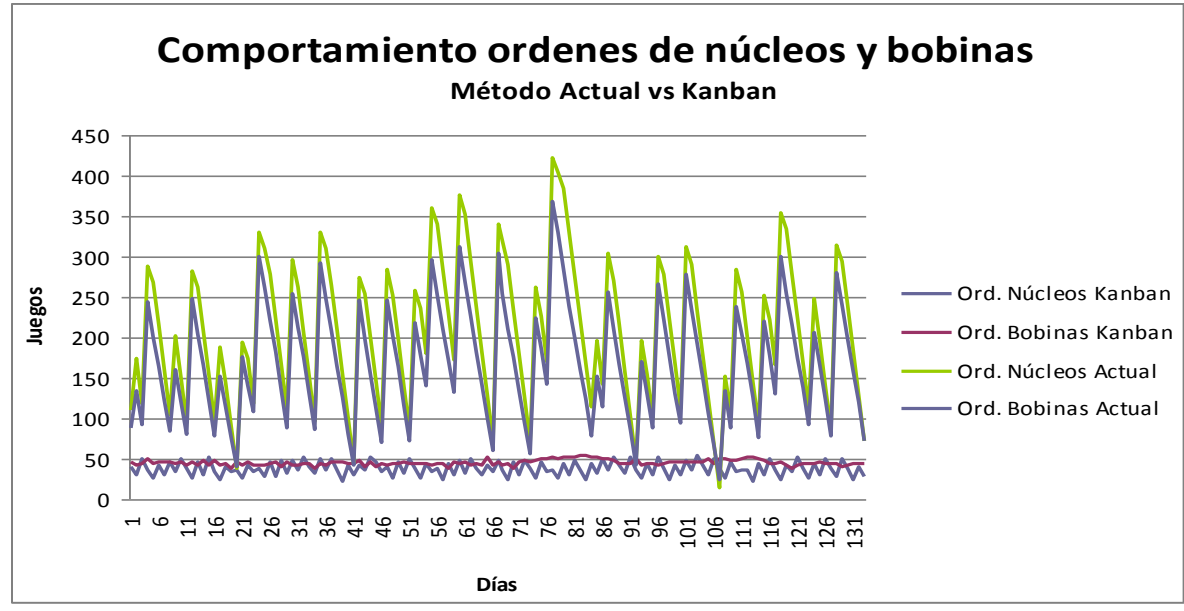

Figura 6. Comportamiento de órdenes de producción de núcleos y bobinas.

Fuente: elaboración propia

En la misma figura 6 se muestra cómo con la metodología Kanban propuesta las órdenes de producción que se generan son empaquetadas en pequeños lotes, facilitándose que la programación inicial de producción para el proceso de núcleo sea anterior a la programación del proceso de bobinado, debido a que los tiempos de producción de núcleo son superiores al tiempo de ciclo de fabricación de bobinado. De esta manera, el centro de trabajo de núcleos da el inicio de marcha para la línea de fabricación de bobinado, evitando que se produzca más de lo que se requiere en el turno posterior en la sección de bobinado.

La tabla 2 presenta el promedio de las cantidades enviadas a producción de bobinas y núcleos para el proceso actual y mejorado con la metodología Kanban, donde se observa la reducción importante en las cantidades de fabricación para las bobinas y los núcleos. Además de la figura 6 y de la tabla 2 se observa cómo se disminuye la variabilidad en estas cantidades a producir, lo que permite realizar mejores procesos de planificación de la producción para operaciones futuras, así como disminuir el inventario de producto en proceso a largo del período de fabricación. 
Tabla 2. Cantidades ordenadas a producción.

\begin{tabular}{|l|c|c|c|c|}
\hline \multirow{2}{*}{ Proceso } & \multicolumn{2}{|c|}{ Cantidad a producir en núcleos } & \multicolumn{2}{c|}{ Cantidad a producir en bobinas } \\
\cline { 2 - 5 } & Media & Desviación & Media & Desviación \\
\hline Actual & 147 & 71 & 183 & 94 \\
\hline Con Kanban & 42 & 6 & 45 & 4 \\
\hline Diferencia & 105 & 65 & 138 & 90 \\
\hline
\end{tabular}

Fuente: elaboración propia

\section{CONCLUSIONES}

La metodología Kanban permite disminuir los niveles de inventario de productos en proceso en líneas de producción de empresas manufactureras, como resultado de producir únicamente lo necesario en aquellos procesos en que esta metodología es aplicada. En este artículo se presentó la aplicación de Kanban en una empresa del sector eléctrico, encontrándose reducciones en el número y en la variabilidad de productos en procesos (bobinas sin núcleo y de núcleo sin bobinas), así como la reducción del número de órdenes enviadas a producción para la fabricación de transformadores de potencia.

La metodología Kanban permite sincronizar las etapas de producción y ensamble en plantas de manufactura, mediante un cambio en la forma de realizar y organizar el trabajo, para lo cual no se hacen necesarios cambios de infraestructura ni tecnología física. El único requisito para la implementación de esta metodología es la formación de personal en la metodología Kanban y la organización de los procesos.

La simulación de procesos de manufactura permite realizar una modelación que se aproxima a la realidad de dichos procesos, con lo cual es posible analizar el desempeño tanto de condiciones actuales, como futuras donde se proponen cambios. A partir de estos análisis de simulación se pueden tomar decisiones que permiten tener mayor certeza sobre los resultados esperados. En el estudio realizado, se simularon el proceso actual y el proceso con la implementación de la metodología Kanban, para la producción de bobinas y núcleos en la fabricación de transformadores de potencia, permitiendo observar claramente el impacto de implementar dicha metodología, con lo cual se observa una posible mejora en el proceso de fabricación estudiado. 


\section{REFERENCIAS}

[1] Arango MD et al. "Reingeniería de procesos y Transformación Organizativa". Editorial Alfaomega. Colombia. 2007.

[2] Gross, J. M., Mcinnis, K.R. “Kanban Made Simple: Demystifying and Applying Toyota's Legendary Manufacturing Process. AMACOM. 2003.

[3] Gstettner, S. and H. Kuhn "Analysis of production control systems Kanban and CONWIP", International Journal of Production Research 34, pp. 3253-3273. 1996.

[4] Imai, M. "Como implementar el Kaizen en el sitio de trabajo (gemba)", McGraw Hill. 1998.

[5] JIPM “Japon Institute of Plant Maintenance". Disponible en http://www.jipm.or.jp/en/home. Consultado el 15 de febrero de 2010.

[6] Japan Management Association. "Kanban Just-In-Time at Toyota: Management Begins at the Workplace". Productivity Press; Rev Sub edition. 1989.

[7] Management Developing Center, S. A. de C. V. "Herramientas de Mejora de Procesos Kaizen, Smed, Kanban, Poka Yoke.” Pp. 44. 2006.

[8] Shimbun, N.K. "Poka-Yoke: Improving Product Quality by Preventing Defects", Productivity Press. 1988.

[9] Shingo, S. "Una revolución en la producción: el sistema SMED. Preparaciones rápidas de máquinas: el sistema SMED”. TGP Hoshin. 2001.

[10] Raymond S. "Custom Kanban: Designing the System to Meet the Needs of Your Environment". Productivity Press; 1 edition. 2006.

[11] SIMUL8, “Control Rules Examples". http://www.simul8.com/support/learning/cgi-bin/learning.cgi?selectedpage $=$ logic \& formtext=logic.htm. Consultada entre enero y febrero de 2010.

[12] Acevedo J. y Urquiaga, A. y Gómez, M. “Gestión de la Cadena de suministro. Centro de estudio de Tecnología de Avanzada (CETA) y Laboratorio de Logística y Gestión de la Producción (Logespro)". Ciudad Habana. 2001.

[13] Geek with Laptop. "El método Toyota aplicado al software”. Disponible en http://bosqueviejo. net/wordpress/2009/06/22/kanban-el-metodo-toyota-aplicado-al-software, Consultada en septiembre de 2009.

[14] Mitra, D. y Mitrani. I. "Analysis of a Kanban discipline for cell coordination in production lines”. I, Management Science 36, 1548-1566. 1990.

[15] Ballesteros, D. "A practical form to apply the System Kanban in the Colombian Mypimes". Scientia et Technica Año XIV, N. ${ }^{\circ}$ 39. 2008.

[16] GS1. "EPC Código Electrónico de Producto". Disponible en: www.gs1co.org Consultado el 28 de septiembre de 2010.

[17] Quesada, X. Kanban Boards. Disponible en: www.xqa.com.ar . consultada el 14 de enero de 2010 